\title{
Hector SLAM 2D Mapping for Simultaneous Localization and Mapping (SLAM)
}

\author{
${ }^{1}$ Shahrizal Saat, ${ }^{1}$ AN.MF. Airini, ${ }^{2}$ Muhammad Salihin Saealal, \\ ${ }^{1}$ A.R. Wan Norhisyam and ${ }^{1}$ M.S. Farees Ezwan \\ ${ }^{1}$ Department of Electronic and Computer Engineering, Faculty of Engineering Technology, \\ Durian Tunggal Malaysia \\ ${ }^{2}$ Department of Electrical Engineering, Faculty of Engineering Technology, \\ Universiti Teknikal Malaysia Melaka (UTeM), Durian Tunggal, Malaysia \\ shahrizal@utem.edu.my
}

\begin{abstract}
This study presents an application of LiDAR sensor for 2D mapping construction in an unkwown environments and capability to localize its own location based on landmark detected. Previously, there are various research actively conducted by others researchers for SLAM application. In general, it can be categorized based on three different type of sensor measurement and technique such as vision based SLAM, RGB-D based SLAM and also laser based SLAM. The main focus in this project is to present an experiment result conducted of a Simultaneous Localization and Mapping (SLAM) application based on laser sensor which is LiDAR in term of capability of mapping construction and localization it self. LiDAR sensor is put on the vehicle that will operate in real-world environments and computational processing done by using Robotic Operating System (ROS). This project is tested and verified in a curtain room with several parameter by using Robot Operating System (ROS). SLAM was implemented to provide localization estimates in environments where there are static landmarks that are only rarely recognized by the vehicle or robot. This project also, consider the features that enter and leave the environment as temporary landmarks that can be used in combination with the rarely seen static landmarks. As conclusion, performance of SLAM by using LiDAR sensor can be apply for several robotic system such as flight control, obstacle avoidance, navigation and other function in the future application.
\end{abstract}

Key words: Simultaneous Localization and Mapping (SLAM), RPLiDAR A2, Robotic Operating System (ROS), computational, landmarks, flight control

\section{INTRODUCTION}

Simultaneously Localization and Mapping (SLAM) refer to the issue that try to localize itself and at the same time with respect to the environment mapping structure. Depending on this situation can be achieved in many ways. The SLAM system means a set of algorithms that work to solve simulation and simultaneous mapping problems (Thrun and Leonard, 2008).

Different type of sensor applied for SLAM will generate variation cumulative errors when mobile robot try to estimate the motion or location (Huang et al., 2013). The biggest challenge in SLAM is to determine whether sensor measurement taken data at the same points in the time comparing to the same object in actual environment (Wang and Slam, 2016).

In this project, utilization of ROS (Robot Operating System) which is a system that controls robotic components from a computer. The ROS system consists of several free nodes, each communicating with other nodes using a subscription/subscription order model. The Robot Operating System (ROS) is a flexible framework for writing robot software. It is a collection of tools, libraries and conventions aimed at facilitating the task of creating robust and robust robot behavior across various robots platforms. The ROS is built from below to promote the development of robotic collaboration software.

Various type of 2D SLAM algorithm available such as Hector SLAM, Gmapping, KartoSLAM, CoreSLAM and LagoSLAM. In this project, performance of Hector SLAM have been evaluated. HectorSLAM combines a 2D SLAM system based on robust scan matching technique. Estimation of robot movement in real time and different parameter of scanning rate from LiDAR sensor tested in this experiment (Beinschob and Reinke, 2015).

In this project, RPLiDAR A2 Laser Scanner with features $360^{\circ} 2 \mathrm{D}$ lidar has been used. It adopts a low price laser triangulation system developed by SLAMTEC and

Corresponding Author: Shahrizal Saat, Department of Electronic and Computer Engineering, Faculty of Engineering Technology, Durian Tunggal, Malaysia shahrizal@utem.edu.my 


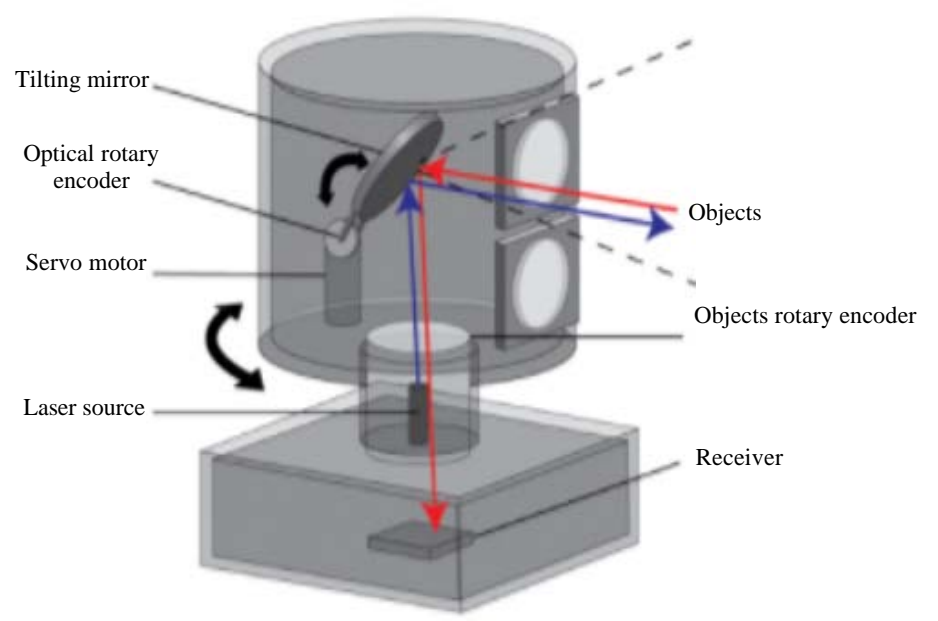

Fig. 1: LiDAR sensor

therefore has a high quality overall performance in all types of enclosed environments and outdoor environments without direct daylight exposure.

Hector SLAM is considered state, if the art for particle filter-based mapping. This SLAM algorithm that can be used without odometer as well as on platforms that exhibit roll or pitch motion of the sensor. While the system does not provide explicit loop closing ability, it is sufficiently exactly for many real world scenarios (Digani et al., 2015).

It can take up to 4000 laser samples by the second with excessive rotation velocity of 600 RPM. RPLiDAR A2 is aimed at SLAMTEC's patented OPTMAG technology, it poses a problem of traditional LiDAR system life in a good way to work steadily for the long term. Figure 1 shows how LiDAR sensor main components.

\section{MATERIALS AND METHODS}

To control the system, this project used ROS that integrate from several nodes. Laser scanner data that takes care of communication with the RPLiDAR laser scanner that allows the vehicle to observe the direction. The laptop will display the map and localize the vehicle from SLAM node and data laser scanner nodes. Figure 2 show overall block diagram of the system.

Software development: This software was used in order to process the analysis and communicate with the hardware. The main software of this project is Robot Operating System (ROS) to control robotic component from a laptop. Linux OS on Ubuntu desktop is required to run the ROS. ROS uses the OS's process management system, user interface, file system and programming utilities. The mapping will show in RViz application show in Fig. 3.

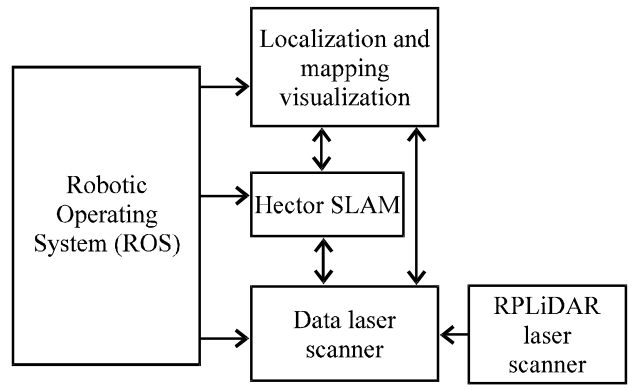

Fig. 2: Overall block diagram of the system

Robotic Operating System (ROS) is a system for controlling robotic components from computer. ROS system comprised from a number of independent nodes, each of which communicates with the other nodes using publish/subscribe messaging model. ROS is a flexible frame work for writing robot software. It is a collection of tools, libraries and conventions that aim to simplify the task of creating complex and robust robot behavior across a wide variety of robotic platforms. ROS was built from the ground up to encourage collaborative robotics software development.

Hardware development: Figure 4 show hardware component involve for this project. Basic ground vehicle used to move the system around known environment in order to evaluate performance of mapping construction and localization based on Hector SLAM in ROS.

Experiment tested by evaluate mapping construction quality in known environment with several parameter adjustment such as scanning frequency of LiDAR sensor. Ability to localize its current location after movement also has been observed. All this experiment done by applying Hector SLAM algorithm in ROS. 


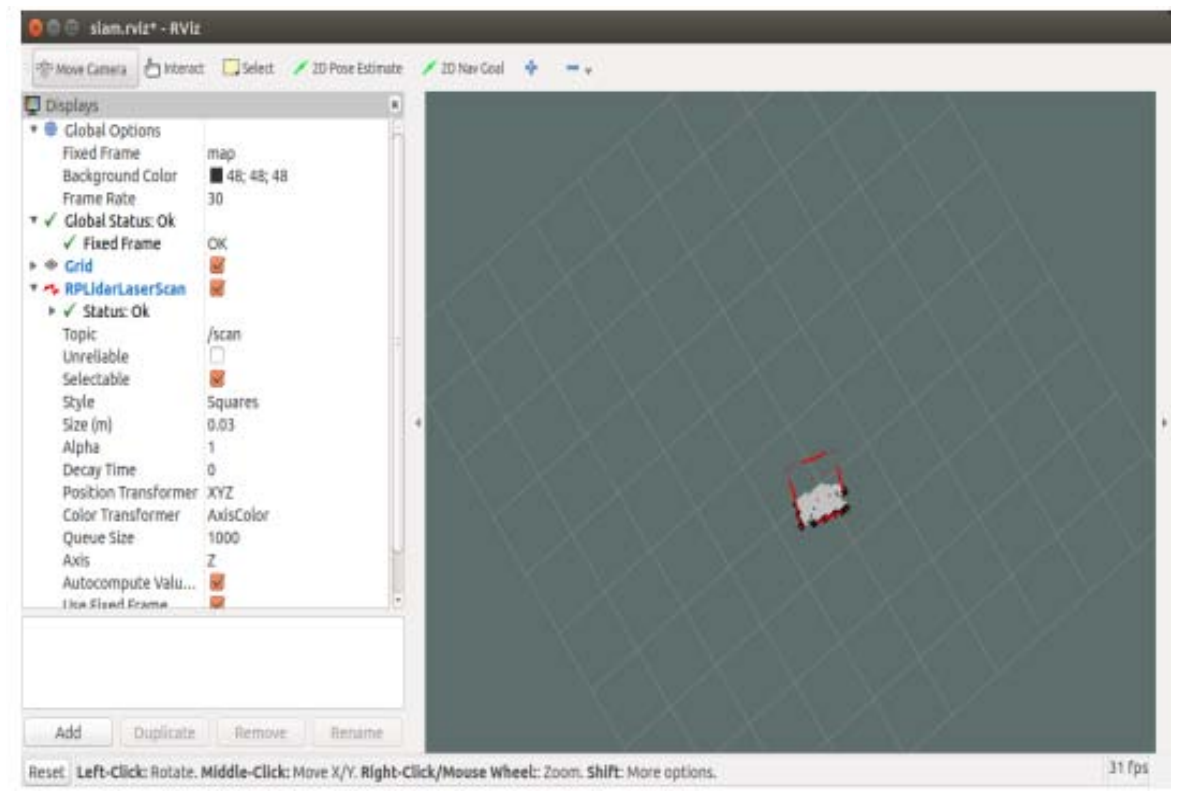

Fig. 3: RViz application

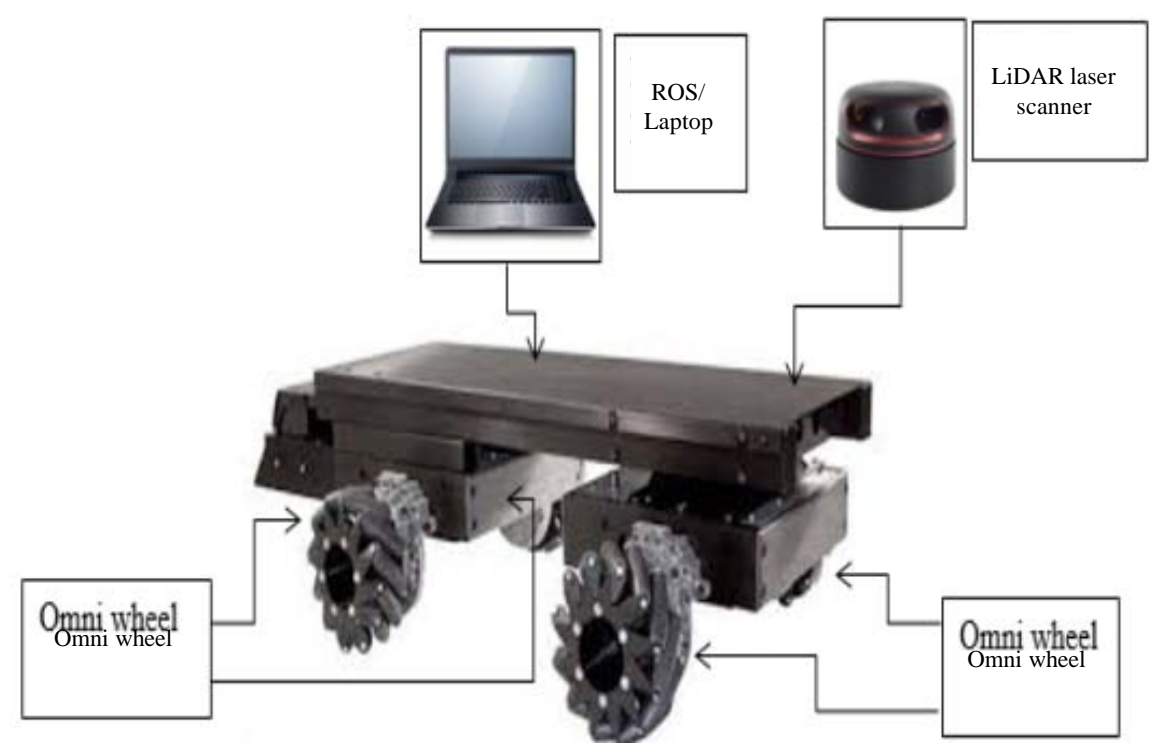

Fig. 4: Hardware components

\section{RESULTS AND DISCUSSION}

Figure 5 show a known environment during performance evaluation of Hector SLAM in ROS by feeding data from RPLiDAR A2 sensor. Scanning frequency has been adjust. The frequency of RPLiDAR scanning rate has been adjust for several parameter and a results obtain as shown in Table 1 .

Next, the system has been test in small indoor environment with several closed space. Based on the previous result obtain, the system has been tested at frequency $21.6 \mathrm{~Hz}$ RPLiDAR scanning rate. Figure 6 show small indoor environment and Fig. 7 show mapping obtain from the test.

Next, the system has been tested at large indoor environment with several closed space. The system also, tested at frequency $21.6 \mathrm{~Hz}$ RPLiDAR scanning rate. Figure 8 show layout plan for large indoor environment and Fig. 9 show results obtain from the test. 


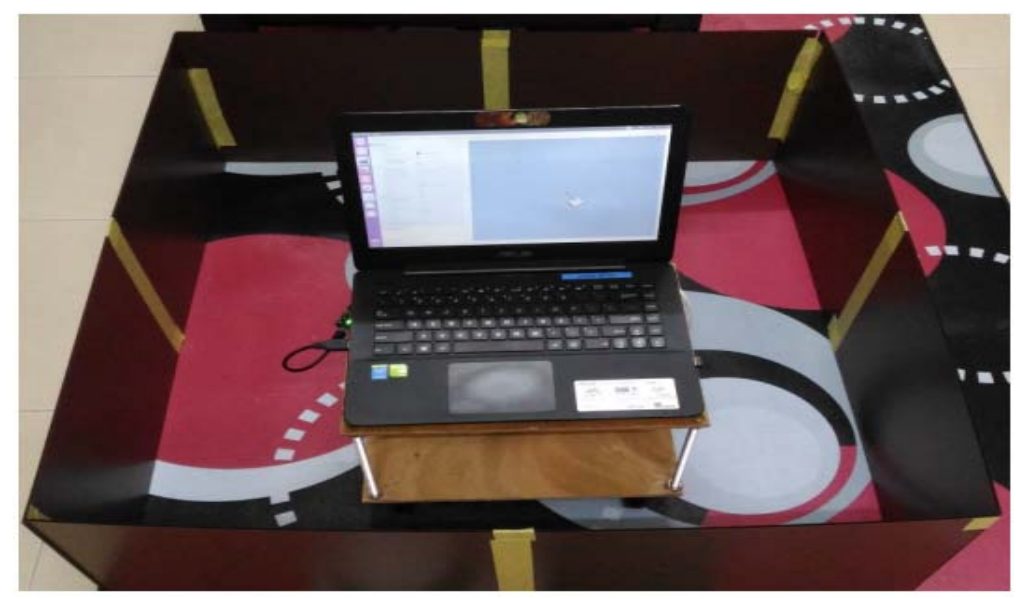

Fig. 5: Known environment test

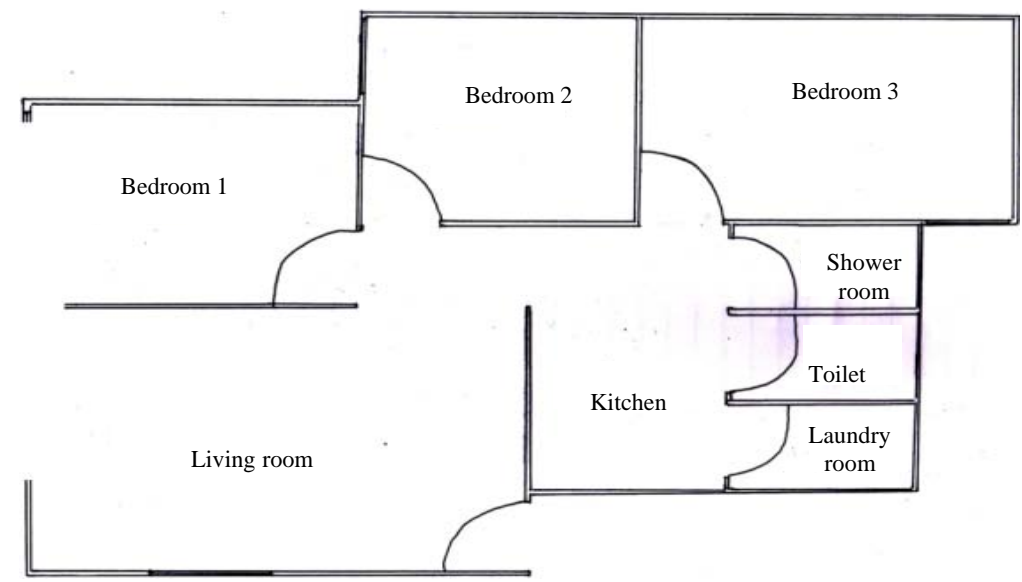

Fig. 6: Layout plan for small indoor environment

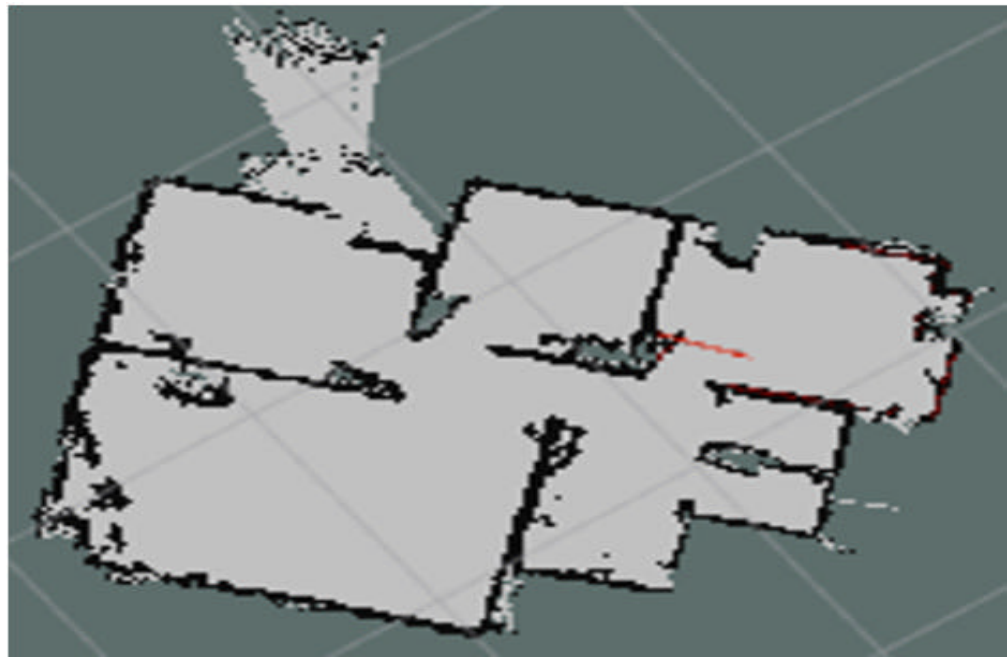

Fig. 7: Mapping construction 


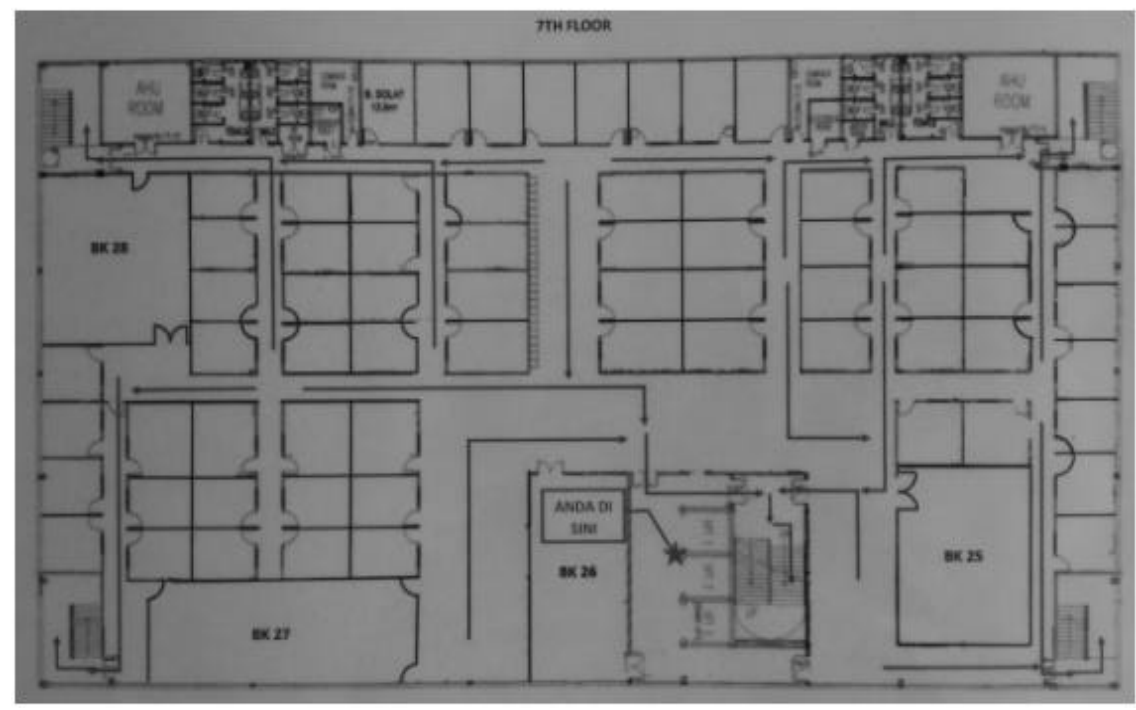

Fig. 8: Layout plan for large indoor environment

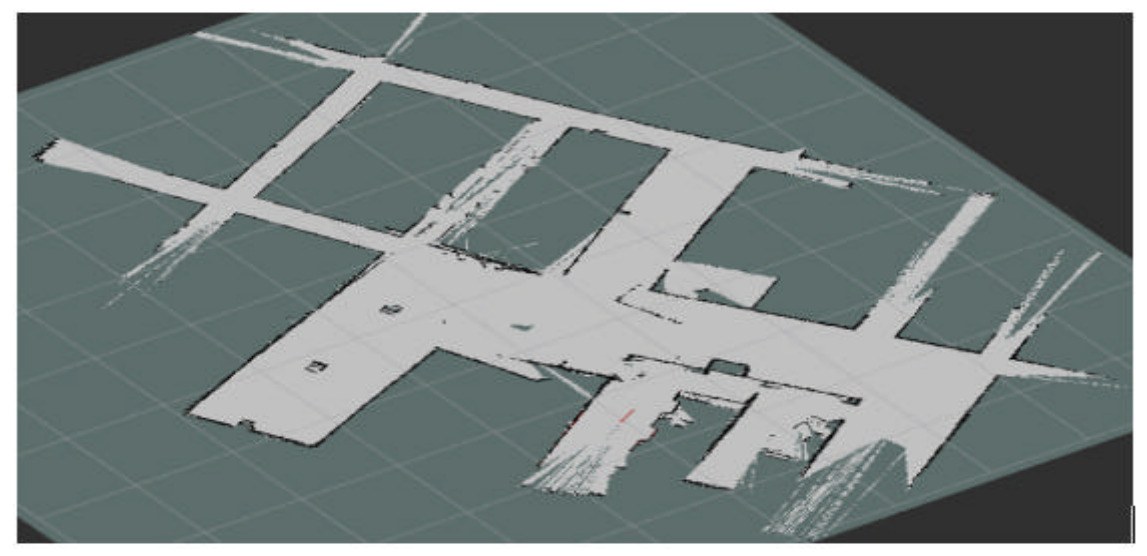

Fig. 9: Mapping construction; Results obtain from the test

Table 1: Mapping construction at different scanning frequency

\begin{tabular}{lll}
\hline Frequency and RPM & Frame grabber & RViz \\
\hline 48 (ADC) & \\
$2.0 \mathrm{~Hz}$ &
\end{tabular}

117 RPM

252 (ADC)

$4.1 \mathrm{~Hz}$

$243 \mathrm{PM}$

456 (ADC)

$8.4 \mathrm{~Hz}$

503 RPM
Table 1: Continue

Frequency and RPM 660 (ADC)

$12.5 \mathrm{~Hz}$

748 RPM

864 (ADC)

$17.5 \mathrm{~Hz}$

1052 RPM

1023 (ADC)

$21.6 \mathrm{~Hz}$

1295 RPM 


\section{CONCLUSION}

Simultaneous Localization and Mapping (SLAM) is a computing problem to build or update an unknown environmental map and at the same time track its own location. In general, Hector SLAM able to construct a good quality of $2 \mathrm{D}$ mapping and localization. However, there are still a huge space can be done in order to improve quality of mapping and localization ability of the system by adding integration with several type of sensor such as Inertial Measurement Unit (IMU) for 3D mapping and navigation system. In the next experiment, others mapping technique such as Gmapping can be test. Practically, we never know the environment but we are always there and only estimate it in the map, hoping to be the same with the environment. This map is built with feature position but often has false readings of features or misidently identifies the same features as two separate. Thus, SLAM approaches consider features that move in the environment.

\section{ACKNOWLEDGEMENT}

The researchers would like to thank for the support given to this research by Ministry of Higher Education Malaysia and Universiti Teknikal Malaysia Melaka(UTeM)underRAGS/1/2015/TK0/FTK/03/B0011 1 research grant.

\section{REFERENCES}

Beinschob, P. and C. Reinke, 2015. Graph SLAM based mapping for AGV localization in large-scale warehouses. Proceedings of the 2015 IEEE International Conference on Intelligent Computer Communication and Processing (ICCP), September 3-5, 2015, IEEE, Cluj-Napoca, Romania, ISBN:978-1-4673-8200-7, pp: 245-248.

Digani, V., L. Sabattini, C. Secchi and C. Fantuzzi, 2015. Ensemble coordination approach in multi-agv systems applied to industrial warehouses. IEEE. Trans. Autom. Sci. Eng., 12: 922-934.

Huang, D., Z. Cai, Y. Wang and X. He, 2013. A real-time fast incremental SLAM method for indoor navigation. Proceedings of the International Conference on Chinese Automation Congress, November 7-8, 2013, IEEE, Changsha, China, ISBN:978-1-4799-0333-7, pp: 171-176.

Thrun, S. and J.J. Leonard, 2008. Simultaneous Localization and Mapping. In: Springer Handbook of Robotics, Siciliano, B. and O. Khatib (Eds.). Springer, Berlin, Heidelberg, Germany, ISBN:978-3-540-23957-4, pp: 871-889.

Wang, K. and A. Slam, 2016. Implementation of odometry with EKF for localization of hector SLAM method. Intl. J. Autom. Smart Technol., 1: 452-456. 